\title{
INSATISFAÇÃO CORPORAL ASSOCIADA A INDICADORES ANTROPOMÉTRICOS EM ADOLESCENTES DE UMA CIDADE COM ÍNDICE DE DESENVOLVIMENTO HUMANO MÉDIO A BAIXO
}

\author{
DRA. ANDREIA PELEGRINI \\ Professora Adjunta do Centro de Ciências da Saúde e do Esporte (CEFID) da \\ Universidade do Estado de Santa Catarina (UDESC), Doutorado em Educação Física pela \\ Universidade Federal de Santa Catarina (CDS/UFSC) \\ (Florianópolis - Santa Catarina - Brasil) \\ e-mail: andreia.pelegrini@udesc.br
}

MS. DIEGO AUGUSTO SANTOS SILVA

Mestre e Doutorando em Educação Física pelo Centro de Desportos da Universidade Federal de Santa Catarina (CDS/UFSC) Bolsista CAPES

(Florianópolis - Santa Catarina - Brasil)

e-mail: diegoaugustoss@yahoo.com.br

\section{MS. ADELSON FERNANDES DA SILVA}

Professor da Universidade Estadual de Montes Claros e Mestre em Avaliação das Atividades Físicas e Desportivas pela Universidade de Trás-os-Montes e Alto Douro

(UTAD - Portugal) (Januária - Minas Gerais - Brasil) e-mail: adelson_fs@yahoo.com.br

\section{DR. EDIO LUIZ PETROSKI}

Professor Titular do Centro de Desportos (CDS) da Universidade Federal de Santa Catarina (UFSC), Doutorado em Educação Física pela Universidade Federal de Santa Maria (UFSM)

(Florianópolis - Santa Catarina - Brasil)

e-mail: petroski@cds.ufsc.br

\section{RESUMO}

O estudo objetivou verificar a associação da insatisfação corporal com indicadores antropométricos em adolescentes. Participaram do estudo 402 adolescentes (14- I7 anos) de Januária-MG. As variáveis coletadas foram: massa corporal, estatura, dobras cutâneas (tríceps, subescapular) e imagem corporal. O IMC e o somatório de duas dobras cutâneas (22DC) foram utilizados como indicadores antropométricos. A insatisfação com a imagem corporal foi de 56,7\%. Observou-se associação da insatisfação corporal com $\Sigma 2 D C$ apenas nos rapazes, 
revelando que àqueles com adiposidade baixa e elevada apresentam, respectivamente, uma probabilidade $26 \%$ e $33 \%$ maior de insatisfação corporal. Conclui-se que a insatisfação corporal é elevada em adolescentes. Além disso, o $\Sigma 2 D C$ se associou a insatisfação corporal no sexo masculino.

PALAVRAS-CHAVE: Adolescentes; antropometria; imagem corporal; insatisfação corporal.

\section{INTRODUÇÃO}

Nas últimas décadas, prevalências elevadas de insatisfação com a imagem corporal em adolescentes têm sido observadas (BYELY et al., 2000; LATZER; TZISCHINSKY; ASAIZA, 2007). Enquanto o sexo feminino busca por um padrão ideal de magreza, o masculino almeja um corpo atlético e musculoso (MCCABE; RICCIARDELLI, 2004).

Estudos internacionais e nacionais (LI et al., 2005; GRAUP et al., 2008; CORSEUIL et al., 2009; PETROSKI; PELEGRINI; GLANER, 2009; PELEGRINI; PETROSKI, 20 I0) apontam que a insatisfação com a imagem corporal atinge mais de $60 \%$ dos adolescentes. A insatisfação corporal elevada pode ser influenciada por aspectos socioculturais (HART, 2003; SMOLAK, 2004), os quais impõem um padrão de beleza nem sempre atingível pelas pessoas. Estas prevalências são preocupantes, principalmente, pela sua relação com os distúrbios alimentares (ALVES et al., 2008).

A associação entre a percepção da imagem corporal com indicadores antropométricos tem sido identificada em inúmeros estudos (PETROSKI; PELEGRINI; GLANER, 2009; PELEGRINI; PETROSKI, 20 I0; CORSEUIL et al., 2009; KAKESHITA; ALMEIDA, 2006; BRANCO; HILARIO; CINTRA, 2006; CONTI; FRUTUOSO; GAMBARDELLA, 2005; VILELA et al., 2004). Entretanto, essa relação permanece não resolvida, pois enquanto alguns estudos sugerem que pessoas com excesso de peso apresentam maior insatisfação com a imagem corporal (CONTI; FRUTUOSO; GAMBARDELLA, 2005; KAKESHITA; ALMEIDA, 2006), outros demonstram que mesmo naquelas com massa corporal adequada, a prevalência de insatisfação é bastante alta (BRANCO; HILARIO; CINTRA, 2006).

A maioria dos estudos, que investigaram a associação entre insatisfação com a imagem corporal e indicadores antropométricos, foi realizada em cidades isoladas da região Sul e Sudeste do Brasil, com alto índice de desenvolvimento humano (IDH). Porém, há escassez de pesquisas em cidades com IDH médio e baixo, o que limita extrapolações acerca desta temática no Brasil. Neste sentido, o presente estudo tem como objetivo verificar a associação entre insatisfação com a imagem e indicadores antropométricos em adolescentes de uma cidade com IDH médio-baixo. 


\section{MATERIAIS E MÉTODOS}

O estudo sobre a associação entre a insatisfação com a imagem corporal e indicadores antropométricos em adolescentes foi desenvolvido a partir de um estudo transversal "Análise da atividade física e aptidão física relacionada à saúde em escolares rurais e urbanos", aprovado por um Comitê de Ética instituicional (Parecer 0 I29/09). O presente estudo foi realizado em uma amostra representativa de adolescentes do município de Januária - MG, situado na região do Médio São Francisco. Esse município é constituído por 67.516 habitantes (BRASIL, 2008). O IDH é de 0,699 e classifica o município com médio-baixo desenvolvimento humano (PROGRAMA DAS NAÇÕES UNIDAS PARA O DESENVOLVIMENTO, 2000).

Para garantir a maior qualidade possível das informaçães obtidas, foi constituída uma equipe de avaliadores, os quais foram treinados e informados sobre os detalhes acerca das variáveis a serem investigadas. Na sequência, realizou-se um estudo piloto com 35 adolescentes de uma escola estadual que não foi selecionada para o trabalho de campo.

O processo de amostragem foi estratificado por escolas públicas do ensino fundamental e médio e conglomerado de turmas. No primeiro estágio, consideraramse somente as escolas que tinham ensino fundamental e médio, pois eram as maiores escolas da região e concentravam a maior quantidade de alunos. Procedeu-se ao sorteio de quais escolas participariam do estudo, tendo como base uma lista fornecida pelas próprias instituições com a idade dos estudantes. No segundo estágio, foram convidados a participar do estudo todos os adolescentes de 14 a 17 anos que estavam presentes em sala de aula no dia da coleta de dados.

Foram calculados vários tamanhos de amostra de acordo com recomendações da literatura (LUIZ; MAGNANINI, 2000), pois este estudo faz parte de uma pesquisa mais ampla, com diferentes desfechos em saúde. Para a presente análise, adotou-se uma prevalência de 65\% (insatisfação com a imagem corporal) (PELEGRINI; PETROSKI, 20 I 0), erro tolerável de cinco pontos percentuais, nível de confiança de $95 \%$, efeito de delineamento de I,5, acrescentando I0\% para possíveis perdas e recusas. Considerando que na região de Januária - MG, 4.495 escolares formavam o ensino fundamental e médio, estimou-se uma amostra de 535 adolescentes. Em virtude das características do processo amostral, que envolveu todos os indivíduos pertencentes aos conglomerados, participaram da amostra 570 adolescentes.

As informações da percepção da imagem corporal foram obtidas com a utilização da escala de silhuetas corporais proposta por Stunkard et al. (1983). O conjunto de silhuetas era mostrado aos adolescentes, e, os mesmos respondiam a duas perguntas: Qual a silhueta que melhor representa a sua aparência corporal 
atual (real)? Qual é a silhueta corporal que você gostaria de ter (ideal)? Quando a variação entre a silhueta real e a ideal foi igual a zero, os adolescentes foram classificados como satisfeitos; e se diferente de zero, insatisfeitos.

Os dados antropométricos (massa corporal - MC, estatura - ES e espessura de dobras cutâneas das regiões do tríceps e subescapular) foram mensurados segundo procedimentos padronizados (CSEP, 2004). Os indicadores antropométricos analisados foram: (i) índice de massa corporal (IMC $=M_{\mathrm{kg}} / \mathrm{ES}_{\mathrm{m}}$ ); (ii) adiposidade corporal por meio do somatório de espessura de duas dobras cutâneas, tríceps e subescapular ( $\Sigma 2 \mathrm{DC})$.

O critério utilizado para a classificação do estado nutricional (IMC) foi proposto por Cole et al. (2002; 2007), recomendado pela International Obesity Task Force (IOTF), baseado nos valores de baixo peso (valores equivalentes ao IMC igual ou inferior a $18,5 \mathrm{~kg} / \mathrm{m}^{2}$ em adultos), sobrepeso (valores equivalentes ao IMC superior que $25 \mathrm{~kg} / \mathrm{m}^{2}$ e inferior que $30 \mathrm{~kg} / \mathrm{m}^{2}$ em adultos) e obesidade (valores equivalente ao IMC igual ou superior que $30 \mathrm{~kg} / \mathrm{m}^{2} \mathrm{em}$ adultos). A adiposidade corporal foi estimada por meio do somatório das dobras cutâneas tricipital e subescapular ( $\left.\sum 2 \mathrm{DC}\right)$, e classificada em: baixa $\left(\sum 2 \mathrm{DC}<13 \mathrm{~mm}\right.$ ou $\left.<15 \mathrm{~mm}\right)$, normal $\left(\sum 2 \mathrm{DC}\right.$ entre 13 e $20 \mathrm{~mm}$ ou entre 15 e $27 \mathrm{~mm}$ ) e elevada ( $\sum 2 \mathrm{DC}>20 \mathrm{~mm}$ ou $>27 \mathrm{~mm}$ ), para rapazes e moças, respectivamente (LOHMAN, 1987).

$\mathrm{Na}$ análise descritiva das variáveis, foram utilizadas médias, desvios padrão e distribuição de frequências. A diferença entre as médias e as proporções de cada variável foi verificada através do teste $U$ de Mann Whitney e do qui quadrado, respectivamente. Ao verificar que a variável dependente (insatisfação com a imagem corporal) apresentou uma prevalência elevada, utilizou-se a regressão de Poisson com ajuste robusto para variância, para examinar as associações entre este desfecho e os indicadores antropométricos (IMC e $\Sigma 2 D C$ ), estimando-se razões de prevalência e os intervalos de confiança. Todas as variáveis foram introduzidas no modelo de regressão ajustado (indicadores antropométricos: IMC e $\Sigma 2 \mathrm{DC}$ ). 0 nível de significância foi estabelecido em 5\% (IC95\%).

\section{RESULTADOS}

Foram excluídos 168 escolares por não responderem completamente o questionário de imagem corporal. Assim, a amostra final foi composta por 405 adolescentes, sendo 167 do sexo masculino e 235 do sexo feminino.

As características gerais da amostra estão apresentadas na Tabela I. Diferenças $(p<0,05)$ entre os sexos foram encontradas nas médias de MC, ES e $\Sigma 2 D C$. Em todas as variáveis, os rapazes apresentaram valores superiores às moças, com exceção do $\Sigma 2 \mathrm{DC}$. 
Tabela I. Características gerais da amostra. Januária, Minas Gerais, 2008.

\begin{tabular}{llll}
\hline Variáveis & Rapazes $(\mathrm{n}=167)$ & Moças $(\mathrm{n}=235)$ & Total $(\mathrm{n}=402)$ \\
\hline MC $(\mathrm{kg})$ & $56,33 \pm 11,17^{*}$ & $51,34 \pm 7,55$ & $53,41 \pm 9,54$ \\
ES $(\mathrm{cm})$ & $167,94 \pm 8,60^{*}$ & $160,51 \pm 6,01$ & $163,60 \pm 8,07$ \\
IMC $\left(\mathrm{kg} / \mathrm{m}^{2}\right)$ & $19,87 \pm 3,17$ & $19,89 \pm 2,48$ & $19,88 \pm 2,78$ \\
$2 \mathrm{DC}(\mathrm{mm})$ & $17,89 \pm 11,17^{*}$ & $26,20 \pm 11,30$ & $22,75 \pm 11,96$ \\
\hline
\end{tabular}

MC: Massa Corporal; ES: Estatura; IMC: Índice de Massa Corporal; 2DC: Somatório de Espessura de Duas Dobras Cutâneas.

* $p<0,05$ para diferença entre os sexos (teste $U$ de Mann Whitney).

A Tabela 2 mostra a proporção de adolescentes classificados segundo os indicadores antropométricos (IMC e adiposidade corporal). Houve associação entre o $\Sigma 2 D C$ e sexo. Estes resultados revelaram maior proporção de adolescentes do sexo masculino nas classificações ideal e baixo, enquanto na classificação alto, proporção mais elevada foi verificada no sexo feminino. Pode-se perceber, ainda, que enquanto na classificação do $\Sigma 2$ DC 23, I \% e 29,6\% dos adolescentes estavam com adiposidade corporal baixa e alta, respectivamente, no IMC, apenas I6,4\% e 8,7\% foram classificados com IMC baixo e alto, respectivamente.

Tabela 2. Distribuição dos adolescentes, por sexo, de acordo com os indicadores antropométricos. Januária, Minas Gerais, 2008.

\begin{tabular}{|c|c|c|c|c|}
\hline Indicadores & Total & Rapazes & Moças & $\mathrm{p}$ \\
\hline antropométricos & $\%(n)$ & $\%(n)$ & $\%(n)$ & \\
\hline IMC & & & & $0,149 *$ \\
\hline Ideal & $74,9(30 \mathrm{I})^{+}$ & $71,9(120)$ & $77,0(|8|)$ & \\
\hline Baixo & $16,4(66)^{\dagger}$ & $16,2(27)$ & 16,6 (39) & \\
\hline Alto & $8,7(35)^{+}$ & $12,0(20)$ & $6,4(15)$ & \\
\hline $2 \mathrm{DC}$ & & & & $<0,\left.00\right|^{*}$ \\
\hline Ideal & $46,0(185)^{\dagger}$ & $37,7(63)$ & $51,9(122)$ & \\
\hline Baixo & $23,1(93)^{+}$ & $40,7(68)$ & $10,6(25)$ & \\
\hline Alto & $30,8(124)^{\dagger}$ & $21,6(36)$ & $37,4(88)$ & \\
\hline
\end{tabular}

IMC: Índice de Massa Corporal; 2DC: Somatório de Espessura de Duas Dobras Cutâneas.

* $p<0,05$ para o teste qui quadrado.

† comparação entre IMC e 2DC (teste para comparação entre duas proporções).

A prevalência de insatisfação com a imagem corporal entre os adolescentes do presente estudo foi de $56,7 \%$, sendo mais elevada no sexo masculino $(63,5 \%)$ 
quando comparado ao feminino (51,9\%; p < 0,05) (dados não apresentados). Nas análises univariadas, é possível observar associação da insatisfação corporal com $\Sigma$ 2DC apenas no sexo masculino (Tabela 3). No modelo ajustado por todas as variáveis (IMC e $\Sigma 2 \mathrm{DC}$ ), a adiposidade corporal permaneceu associada à insatisfação com a imagem corporal, revelando que os rapazes com $\Sigma 2 \mathrm{DC}$ baixo e elevado têm, respectivamente, uma probabilidade $26 \%$ e $33 \%$ maior de apresentar insatisfação com a imagem corporal (Tabela 3).

Tabela 3. Associação da insatisfação com a imagem corporal com os indicadores antropométricos (categoria de referência: satisfeito com a imagem corporal). Januária, Minas Gerais, 2008.

\begin{tabular}{|c|c|c|c|c|}
\hline \multirow{2}{*}{ Indicadores } & \multicolumn{2}{|c|}{ Rapazes } & \multicolumn{2}{|c|}{ Moças } \\
\hline & RP (IC95\%) & RP (IC95\%) & RP (IC95\%) & RP**(IC95\%) \\
\hline \multicolumn{5}{|l|}{ IMC } \\
\hline Ideal & I & I & I & I \\
\hline Baixo & I, I $(0,85-1,61)$ & I,। I (0,9|-I,34) & I,25 (0,94- I,67) & $1,13(0,95-1,36)$ \\
\hline Alto & I,24 (0,95-I,6I) & $0,98(0,70-1,37)$ & I,22 (0,79-1,89) & I,09 (0,82-1,43) \\
\hline \multicolumn{5}{|l|}{ 2DC } \\
\hline Ideal & I & I & I & I \\
\hline Baixo & । ,28 (1,09- I,50)* & I,26 (I,07-1,48)* & I, I I (0,90-I,38) & I,09 $(0,88-1,34)$ \\
\hline Alto & । ,32 (I,09- |,59)* & I,33 (I,02-1,75)* & I,04 (0,9| - I,20) & $1,06(0,91-1,23)$ \\
\hline
\end{tabular}

IMC: Índice de Massa Corporal; 2DC: Somatório de Espessura de Duas Dobras cutâneas. RP: Razão de Prevalência; IC: Intervalo de Confiança.

* $p<0,05$ (regressão de Poisson).

** RP ajustada para todas as variáveis do modelo (IMC e 2DC).

\section{DISCUSSÃO}

O estudo atual investigou a associação entre a insatisfação com a imagem corporal e os indicadores antropométricos (IMC e $\Sigma 2 \mathrm{DC}$ ) em adolescentes de uma cidade com desenvolvimento humano médio-baixo. Os achados demonstraram associação entre a insatisfação com a imagem corporal e $\Sigma 2 \mathrm{DC}$ no sexo masculino. Estes resultados revelaram que os rapazes com $\Sigma 2 \mathrm{DC}$ baixo e elevado têm maiores probabilidades de apresentar insatisfação com a imagem corporal que aqueles com $\Sigma$ 2DC ideal. Ou seja, o indicador antropométrico melhor associado à insatisfação com a imagem corporal é o $\Sigma 2 \mathrm{DC}$. 
Estudo conduzido em adolescentes domiciliados na área rural e urbana de uma cidade de pequeno porte (IDH médio-alto) do estado de Santa Catarina (PETROSKI; PELEGRINI; GLANER, 2009) também verificou que os adolescentes com adiposidade corporal mais elevada apresentaram maior insatisfação com a imagem corporal. Essas evidências sugerem a necessidade de orientações nutricionais, pois adolescentes insatisfeitos com a imagem corporal, frequentemente, adotam comportamentos alimentares inadequados e práticas não saudáveis de redução do peso corporal (VILELA et al., 2004).

Mais da metade dos adolescentes do presente estudo estavam insatisfeitos com a imagem corporal. Vilela et al. (2004), ao investigar a frequência de possíveis transtornos de alimentação e comportamentos alimentares inadequados em crianças e adolescentes (7 a 19 anos) do interior de Minas Gerais (IDH médio), verificaram $59 \%$ de insatisfação corporal. Pesquisa conduzida em crianças e adolescentes ( 9 a 16 anos) de escolas públicas e privadas da cidade de Florianópolis - SC, capital brasileira com o melhor IDH, revelou prevalência de 67,0\% de insatisfação com a imagem corporal (GRAUP et al., 2008). Em outro estudo realizado na capital de Santa Catarina com adolescentes ( 14 a I 8 anos) de escolas públicas, a prevalência de insatisfação corporal foi de 65,5\% (PELEGRINI; PETROSKI, 20I0). Petroski, Pelegrini e Glaner (2009), ao investigar a prevalência de insatisfação com a imagem corporal em adolescentes ( 13 a 17 anos) domiciliados nas áreas urbana e rural do Oeste catarinense e Norte gaúcho, constataram que 63,4\% dos adolescentes estavam insatisfeitos com a imagem corporal. Em estudo internacional (LI et al., 2005) conduzido em crianças e adolescentes ( 3 a 15 anos) chineses, foi encontrada uma prevalência de $59,9 \%$ de insatisfação corporal.

As elevadas proporções de insatisfação com a imagem corporal podem ser explicadas pelo processo de modernização, caracterizado pelas mudanças da vida urbana, cujas pessoas estão adotando comportamentos cada vez mais inadequados de atividade física e hábitos alimentares não saudáveis, os quais contribuem para o incremento do peso corporal e, consequentemente, aumento da insatisfação com a imagem corporal.

Maior proporção de insatisfação com a imagem corporal foi verificada no sexo masculino (63,5\%), quando comparado ao feminino (51,9\%; $p<0,05$ ). Estes resultados corroboram os encontrados em adolescentes de 14 a 18 anos da cidade de Florianópolis - SC (PELEGRINI; PETROSKI, 20I0). A maior prevalência de insatisfação corporal no sexo masculino pode ser explicada pela pressão exercida pela sociedade, principalmente, pela influência da mídia, a qual impõe um corpo musculoso e atlético (MCCABE; RICCIARDELLI, 2004). Por outro lado, o sexo feminino está mais susceptível à adoção de estratégias que visam à redução do peso corporal (MCCABE; RICCIARDELLI, 2004). 
Uma informação interessante encontrada no presente estudo foi que, enquanto no $\Sigma 2 \mathrm{DC}, 29,6 \%$ dos adolescentes foram classificados com adiposidade corporal elevada, no IMC, apenas $8,7 \%$ destes estavam nesta condição. Este indicador (IMC) tem sido questionado quando o seu uso tem a finalidade de verificar os níveis de gordura corporal (TAYLOR et al., 2003), pois o indivíduo pode apresentar um IMC dentro do padrão ideal e, no entanto, possuir uma quantidade de gordura corporal acima do ideal; ou apresentar um IMC abaixo do recomendado e possuir uma quantidade de gordura corporal ideal. Por outro lado, a adiposidade corporal obtida por meio da mensuração de dobras cutâneas tem tido larga aceitação, haja vista a associação com o critério para validade de outras técnicas (pesagem hidrostática) (THORLAND et al., 1984). Em estudo conduzido em adolescentes (I I a 17 anos), foi verificado que o IMC não apresentou consistência para classificar rapazes e moças quanto à gordura corporal, pois somente $49,0 \%$ das moças e $57,3 \%$ dos rapazes foram classificados, concomitantemente, pelo IMC e $\Sigma 2$ DC (GLANER, 2005).

Uma das limitações do presente estudo refere-se a não investigação do status de maturação biológica dos jovens. A faixa etária que eles se encontram é um momento de transição do período pré-púbere para o púbere, e deste, para o pós-púbere. Tais transições refletem na composição corporal e estado nutricional dos jovens como relatado em Silva et al. (20 I 0), e podem exercer influência também na percepção da imagem corporal. Outra limitação do presente estudo refere-se à utilização de desenho bidimensional das silhuetas corporais para a avaliação da imagem corporal. Isto pode implicar falhas na representação total do corpo e/ou na distribuição da massa de gordura. Ademais, esse instrumento não apresenta validação para a população jovem brasileira. Em contrapartida, é interessante destacar que esta foi a primeira investigação a verificar a associação da insatisfação com a imagem corporal, com indicadores antropométricos, em uma amostra representativa de adolescentes de uma cidade com médio-baixo desenvolvimento humano do interior de Minas Gerais.

\section{CONCLUSÕES}

Os achados encontrados no presente estudo permitem concluir que mesmo em cidades com problemas acentuados de distribuição de renda, serviços, educação e saúde, a prevalência de insatisfação com a imagem corporal é elevada entre adolescentes. Além disso, observou-se que o $\Sigma 2 \mathrm{DC}$ foi o indicador antropométrico que apresenta relação mais forte com a insatisfação com a imagem corporal em adolescentes, sobretudo, no sexo masculino.

Neste sentido, percebe-se a necessidade de intervenções voltada à orientação nutricional no ambiente escolar e ao incentivo à prática regular de atividade física, visando à redução do peso corporal e, consequentemente, da insatisfação com a imagem corporal em adolescentes. 
Body dissatisfaction among adolescents from a town with a medium/low human development index

ABSTRACT: The objective of this study was to evaluate the association between body dissatisfaction and anthropometric indicators in adolescents from a town with a medium/low human development index. A total of 402 adolescents (1 4 - 17 years) from the town of Januária, Minas Gerais, participated in the study. The following variables were collected: body weight, height, skinfolds (triceps, subscapular), and body image. BMI and the sum of the two skinfolds (2SF) were used as anthropometric indicators. The prevalence of body image dissatisfaction was 56.7\%. An association between body dissatisfaction and 2SF was only observed in boys, with a $26 \%$ and $42 \%$ higher probability of body dissatisfaction in those presenting low and elevated adiposity, respectively. In conclusion, the body dissatisfaction was high among the adolescents studied. In addition, 2SF was associated with body dissatisfaction in adolescent boys.

KEYWORDS: Adolescents; anthropometry; body image; body dissatisfaction.

\section{Insatisfacción corporal en adolescentes de una ciudad con medio/bajo índice de desarrollo humano}

RESUMEN: El objetivo de este estudio fue verificar la asociación de la insatisfacción corporal con indicadores antropométricos, en adolescentes de una ciudad con medio-bajo índice de desarrollo humano. La muestra comprende 402 adolescentes ( I 4- I 7 años) de Januária-MG. Las variables analizadas son: peso, estatura, pliegues cutáneos (tríceps, subescapular) y la imagen corporal. El IMC y la sumatoria de pliegues cutáneos fueron utilizados como indicadores antropométricos. La insatisfacción con la imagen corporal fue de 56,7\%. Se observó asociación de la insatisfacción corporal y el 2PC, únicamente en adolescentes de sexo masculino. Revelando que los que tienen baja y demasiada grasa en el cuerpo presentaron 26\% y $42 \%$ más de insatisfacción corporal, respectivamente. El estudio concluyó que la insatisfacción corporal es alta en adolescentes. Por otra parte se relaciona el 2PC con la insatisfacción corporal en sexo masculino.

àqueles com adiposidade baixa e elevada apresentam, respectivamente, uma probabilidade 26\% e 33\% maior

PALABRAS CLAVE: Adolescente; antropometría; imagen corporal; insatisfacción corporal.

\section{REFERÊNCIAS}

ALVES, E. et al. Prevalência de sintomas de anorexia nervosa e insatisfação com a imagem corporal em adolescentes do sexo feminino do município de Florianópolis, Santa Catarina, Brasil. Cadernos de Saúde Pública, v. 24, n. 3, p. 503-512, mar. 2008.

BRANCO, L. M.; HILÁRIO, M. O. E.; CINTRA, I. P. Percepção e satisfação corporal em adolescentes e a relação com seu estado nutricional. Revista de Psiquiatria Clínica, v. 33, n. 6 , p. 292-296, 2006. 
BRASIL. Ministério do Planejamento, Orçamento e Gestão. Instituto Brasileiro de Geografia e Estatística (IBGE). Disponível em: <http://www.ibge.gov.br/cidadesat/topwindow.htm? I > . Acesso em: 20 março 2010.

BYELY, L., et al. A prospective study of familial and social influences on girls' body image and dieting. International Journal of Eating Disorders, New York, v. 28, n. 2, p. I55-164, sep. 2000.

CANADIAN SOCIETY FOR EXERCISE PHYSIOLOGY (CSEP). The Canadian Physical Activity, Fitness and Lifestyle Appraisal: CSEP's guide to health active living. 3sd ed. Ottawa: CSEP, 2004.

COLE, T. J., et al. Establishing a standard definition for child overweight and obesity worldwide: International survey. British Medical Journal, London, v. 320, n. 7244, p. I240, may. 2002.

COLE, T.J., et al. Body mass index cut offs to define thinness in children and adolescents: International survey. British Medical Journal, London, v. 335, n. 7612, p. 194, jul. 2007.

CONTI, M. A.; FRUTUOSO, M. F. P.; GAMBARDELLA, A. M. D. Excesso de peso e insatisfação corporal em adolescentes. Revista de Nutrição, Campinas, v. I8, n. 4, p. 49|-497, jul./ago. 2005.

CORSEUIL, M. W., et al. Prevalência de insatisfação com a imagem corporal e sua associação com a inadequação nutricional em adolescentes. Revista da Educação Física/UEM, Maringá, v. 20, n. I, p. 25-31, 2009.

GLANER, M. F. Aptidão física relacionada à saúde de adolescentes rurais e urbanos em relação a critérios de referência. Revista Brasileira de Educação Física e Esporte, São Paulo, v. 19, n. I, p. 13-24, mar. 2005.

GRAUP, S., et al. Associação entre a percepção da imagem corporal e indicadores antropométricos de escolares. Revista Brasileira de Educação Física e Esporte, São Paulo, v. 22, n. 2 , p. 129-138, abr./jun. 2008.

HART, E. A. Avaliando a imagem corporal. In: TRITSCHLER, K. Medida e avaliação em Educação Física e esportes de Barrow \& McGee. I. ed. Barueri: Manole, 2003. p. 457-488.

KAKESHITA, I. S.; ALMEIDA, S. S. Relação entre índice de massa corporal e a percepção da autoimagem em universitários. Revista de Saúde Pública, São Paulo, v. 40, n. 3, p. 497-504, jun. 2006.

LATZER, Y.; TZISCHINSKY, O.; ASAIZA, F. Disordered eating related behaviors among Arab schoolgirls in Israel: An epidemiological study. International Journal of Eating Disorders, New York, v. 40, n. 3, p. 263-270, apr. 2007.

LOHMAN, T.G. The use of skinfold to estimate body fatness on children and youth. Journal of Physical Education, Recreation \& Dance, Reston, v. 58, p. 98- I03, nov./dec. 1987. 
LI, Y., et al. Body image perceptions among Chinese children and adolescents. Body Image, Amsterdam, v. 2, n. 2, p. 9|-103, jun. 2005.

LUIZ. R. R.; MAGNANINI, M. M. F. A lógica da determinação do tamanho da amostra em investigações epidemiológicas. Cadernos de Saúde Coletiva, Rio de Janeiro, v. 8, n. 2, p. 9-28, ago./dez. 2000.

MCCABE, M. P.; RICCIARDELLI, L. A. Body image dissatisfaction among males across the lifespan: a review of past literature. Journal of Psychosomatic Research, London, v. 56, n.6, p. 675-685, jun. 2004.

Instituto Nacional de Estudos e Pesquisas Educacionais - INEP - Censo Educacional. Disponível em: <http://www.ibge.gov.br/cidadesat/topwindow.html>. Acesso em: 10 março 2010.

PELEGRINI, A.; PETROSKI, E. L. The association between body dissatisfaction and nutritional status in adolescents. Human Movement, Warsaw, v. II, n. I, p. 5I-57, jun. 2010.

PETROSKI, E. L.; PELEGRINI, A.; GLANER, M. F. Insatisfação corporal em adolescentes rurais e urbanos. Motricidade, Vila Real, v. 5, n. 4, p. 13-25, dez. 2009.

PROGRAMA DAS NAÇÕES UNIDAS PARA O DESENVOLVIMENTO (PNUD). Ranking do Índice de Desenvolvimento Municipal dos municípios do Brasil. Disponível em: < http:// www.pnud.org.br/atlas/tabelas/index.php>. Acesso em: I 5 set. 2009.

Índice de massa corporal: um questionamento baseado em evidências. Arquivos Brasileiros de Cardiologia, Rio de Janeiro, v. 79, n. I, p. 6I-69, jul. 2002.

SILVA, D.A.S., et al. Comparação do crescimento de crianças e adolescentes brasileiros com curvas de referência para crescimento físico: dados do Projeto Esporte Brasil. Jornal de Pediatria, Rio de Janeiro, v. 86, n. 2, p. I I5-120, mar/abr. 2010.

SMOLAK, L. Body image in children and adolescents: where do we go from here? Body Image, Amsterdam, v. I, n. I, p. I5-28, jan. 2004.

STUNKARD, A. J.; SORENSON, T.; SCHLUSINGER F. Use of the Danish adoption register for the study of obesity and thinness. In: KETY, S. S.; ROWLAND, L. P.; SIDMAN, R. L.; MATTHYSSE, S. W. The genetics of neurological and psychiatric disorders. New York, NY: Raven, 1983. p. I15-120.

TAYLOR, R. W., et al. Identifying adolescents with high percentage body fat: a comparison of BMI cutoffs using age and stage of pubertal development compared with BMI cutoffs using age alone. European Journal of Clinical Nutrition, London, v. 57, n. 6, p. 764-769, jun. 2003.

THORLAND, W. G., et al. Estimation of body density in adolescent athletes. Human Biology, Detroit, v. 56, n. 3, p. 439-448, sep. 1984. 
VILELA, J. E. M. et al. Transtornos alimentares em escolares. Jornal de Pediatria, Porto Alegre, v. 80, n. I, p. 49-54, jan./fev. 2004.

Recebido: 30 jun. 2010

Aprovado: 04 jan. 2011

Endereço para correspondência: Andreia Pelegrini Universidade do Estado de Santa Catarina - UDESC Centro de Ciências da Saúde e do Esporte - CEFID. Departamento de Educação Física. Rua Pascoal Simone, 358

Bairro Coqueiros Florianópolis-SC CEP 88080-350 